\title{
Los medios de comunicación como recurso para formar en competencias en el área de la Economía Financiera
}

Alfredo Juan Grau Grau ${ }^{\mathrm{b}}$ y Federico Ramírez López

aE-mail: Alfredo.Grau@uv.es, Departamento Finanzas Empresariales (Facultat d'Economía-UV),

bE-mail: Federico.Ramirez@uv.es, Departamento Finanzas Empresariales (Facultat d'Economía-UV).

\begin{abstract}
The aim of this study is that our students are competent in the field of Finance once completed their studies and are able to function in future executive responsibilities. We implement cooperative work methodology that allows us to develop a teaching focused on student participation by encouraging the development of deeper knowledge.

The evaluation results show that the level of development of financial skills show quite satisfactory levels. The results of academic performance compared to previous years also show a much higher average.
\end{abstract}

Keywords: media, professional skills, cooperative working methodology, indebtedness policy, higher education.

\begin{abstract}
Resumen
El objetivo que nos planteamos en este trabajo es que nuestros estudiantes sean competentes en el ámbito de las Finanzas una vez finalizado sus estudios, y sean capaces de desenvolverse con soltura en el cargo ejecutivo donde desempeñen sus funciones directivas. Para ello, implementamos la metodología de Trabajo Cooperativo que nos permite desarrollar una enseñanza centrada en la participación del alumnado favoreciendo el desarrollo de un conocimiento más profundo.

Los resultados de evaluación revelan que el nivel de desarrollo de las competencias profesionales de talante financiero, arrojan niveles bastante satisfactorios. Los resultados del rendimiento académico respecto a cursos anteriores muestran también una media mucho más elevada.
\end{abstract}

Palabras clave: medios de comunicación, competencias profesionales, metodología de trabajo cooperativo, politicas de endeudamiento, educación superior. 


\section{Introducción}

En las décadas precedentes se ha observado que los medios de comunicación han ido creciendo de forma exponencial, ejerciendo una influencia directa sobre el comportamiento de la sociedad en su conjunto. Centrándonos en el contexto académico, y tal y como asevera Arnoldi (2007), estos medios de comunicación también han sido notablemente influyentes en nuestros estudiantes a la hora de transmitir la información y lo que es más relevante, la transmisión del conocimiento.

Gran parte de la producción científica intrínsecamente relacionada con este fenómeno, por ejemplo, Ricoy (2006) y Buil et al. (2013), señalan que los medios de comunicación pueden ser utilizados al servicio de la enseñanza para desarrollar y fomentar la capacidad analítica, reflexiva y crítica.

Estos argumentos de partida nos alientan a creer que el uso correcto y adecuado de este medio podrían ser una buena herramienta no solo para desarrollar estas capacidades sino también, para desarrollar las competencia profesionales del conjunto de nuestro alumnado.

Estas herramientas educativas han sido objeto de todo tipo de críticas. Éstas vendrían justificadas por el hecho de que la información podría venir sesgada por los individuos que las emiten, bien por cuestiones económicas, religiosas o políticas. Por otro lado, no han sido pocos los investigadores que han ensalzado sus bondades. De Arias (2005) indica que la información que se genera es un buen recurso para estar al día de temas de candente actualidad, con contenidos muy variados, y además acerca a los individuos a la problemática de la vida real. Por otro lado y en la misma línea, Cabero y Guerra (2011) afirman que estos recursos son altamente didácticos y por ende podrían desarrollar las competencias informativas, el espíritu crítico y la opinión reflexiva.

En el presente estudio hemos seleccionado la información contenida en la prensa gráfica y escrita como recurso educativo. Este medio ha adquirido un especial interés (McClune y Jarman, 2010) en los últimos años ya que las nuevas tecnologías han facilitado el rápido acceso, por ejemplo, la prensa on-line, teléfono móvil, tablets, portátiles, etc. Adicionalmente estos autores resaltan la gran capacidad de estos dispositivos para actualizar la información en contraposición a la información contenida en los libros académicos.

Las instituciones educativas también han realizado un especial esfuerzo para que los estudiantes universitarios se titulen con un nivel de competencias profesionales que les permita enfrentarse al mundo laboral. A tal fin el EEES (Espacio Europeo de Educación Superior) pone al servicio de la docencia un conjunto de recomendaciones para que la Educación Superior pueda alcanzar este objetivo.

Al hilo de lo anterior Gutiérrez et al. (2013), entre otros, resaltan que el nivel competencial tienen que tener como finalidad primordial que los estudiantes sepan interpretar y evaluar la información de forma adecuada, así como ser capaces de generar nueva información y tomar decisiones; es decir, desarrollar un pensamiento comprensivo, crítico y creativo. 


\section{Objetivos}

Los medios de comunicación de forma continua proporcionan información asociada al mundo empresarial y sobre todo, relacionadas con la Economía Financiera. En este trabajo pretendemos hacer uso de la información relevante directamente relacionada con la realidad de las empresas cotizadas en el mercado de capitales español. Desarrollamos esta experiencia en innovación educativa sobre los alumnos matriculados en el Máster de Dirección de Empresas (MBA) de la Facultad de Economía en la Universidad de Valencia.

El objetivo general que nos planteamos es que una vez que nuestros estudiantes han finalizado el máster sean capaces de desenvolverse con soltura en el cargo directivo donde desempeñará sus funciones directivas. En este caso concretamente pretendemos que desarrollen las competencias directamente relacionadas con cuestiones de talante financiero. Para ello, aplicaremos una metodología de Trabajo Cooperativo que más adelante explicaremos y desarrollaremos.

Los objetivos más concretos que pretendemos alcanzar serían:

- Estrechar la brecha existente entre los conocimientos adquiridos por el alumnado y su correspondiente aplicación a las noticias publicadas en la prensa gráfica relacionadas con el día a día de los mercados financieros.

- Fomentar el pensamiento crítico a colación de los contenidos publicados en las noticias de talante financiero.

- Promover el gusto por estar siempre informado y al día de las noticias financieras publicadas en medios fiables y que las abordan con rigor.

\subsection{Aprendizaje vs. Competencias Profesionales}

Layer (2004) comprueba que en estos últimos años las universidades manifiestan un destacado interés en la relación existente entre la Educación Superior y la inserción de sus titulados en el mundo laboral. Semeijn et al. (2005) advierte que todo esto le confiere al sistema universitario la gran responsabilidad a la hora de formar adecuadamente a su alumnado, para que en un futuro puedan desenvolverse de forma adecuada en sus puestos de trabajo.

Las competencias educativas por un lado, robustecen las posibilidades de lograr un trabajo que no tiene por qué estar estrechamente asociado al área formativa, por otro lado refuerzan las posibilidades de que puedan desarrollar de forma óptima las exigencias del puesto a ocupar (Heijke et al., 2003).

Las competencias a las que hacemos alusión y que pretendemos desarrollar en nuestros alumnos a través de la experiencia implementada son:

- Que los estudiantes sepan aplicar sus conocimientos a su trabajo o vocación de una forma profesional y posean las competencias que suelen demostrarse por medio de la elaboración y defensa de argumentos y la resolución de problemas dentro de su área de estudio (CB2) .

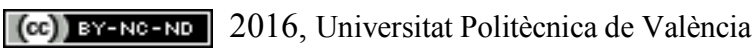


- Habilidad para analizar y buscar información proveniente de fuentes diversas (CG6).

- Capacidad para trabajar en equipo (CG12).

- Capacidad de aprendizaje autónomo (CG17).

- Ser capaz de elaborar e interpretar la información contable, tanto la financiera, destinada a los usuarios externos, como la interna, destinada al control de gestión y la toma de decisiones (CE2).

- Ser capaz de resolver problemas de valoración financiera, tanto en decisiones de financiación, como de inversión empresarial, en el entorno nacional e internacional (CE4).

- Conocer y analizar los mercados financieros, así como las operaciones financieras anejas al ámbito empresarial (CE8).

- Conocer los fundamentos básicos que rigen las operaciones y los mercados financieros (CE49).

- Capacidad para medir y valorar el riesgo de los activos financieros y sus aplicaciones en las inversiones productivas (CE52).

- Conocer los diferentes instrumentos de financiación y ser capaz de determinar la política de endeudamiento de la empresa (CE56).

\section{Desarrollo de la innovación}

\subsection{Metodología de Trabajo Cooperativo}

La metodología seleccionada para desarrollar la innovación será la de Trabajo Cooperativo que dadas sus características, que pasamos a explicar, se ajustan perfectamente a nuestros propósitos. La principal ventaja de este método es que nos permite desarrollar una enseñanza centrada en la participación del alumnado donde la responsabilidad del aprendizaje depende estrechamente de su nivel de implicación y compromiso. Ello conduce a adquirir unos conocimientos con mayor profundidad y en consecuencia, favorecen su durabilidad a lo largo el tiempo y su aplicabilidad a distintos campos y situaciones.

Ariza (2000) afirma que esta metodología se caracteriza por fomentar la adquisición de conocimientos, habilidades o actitudes como resultado del trabajo en grupo o, en otras palabras, un aprendizaje individual como resultado de un proceso grupal.

El cometido del profesor será la de mediador entre los distintos integrantes del grupo así como velar para que el transcurso de la actividad sea de forma satisfactoria. Se le confiere también la responsabilidad de promover la capacidad de reflexión en sentido constructivo a la par que crítico. El desarrollo de las Tecnologías de la Información y la Comunicación (TIC) aportan un gran valor al desarrollo de la actividad ya que, facilita la manipulación e interpretación de la información utilizada. Por otra parte, la UV pone a disposición de la comunidad educativa una plataforma virtual que permite publicar e interactuar a los distintos miembros vinculados con la actividad, esto es, docentes y discentes.

(cc) EY-NC-ND 2016, Universitat Politècnica de València 
Tal y como afirman, entre otros, Sáez y Ruiz (2012) y Muñoz et al. (2014) las TIC son imprescindibles en la sociedad actual para trasmitir el conocimiento y además, gozan de gran aceptación entre los profesores y los estudiantes.

\subsection{Diseño de la innovación}

El diseño de la innovación se vertebra a través del uso de la metodología de Trabajo Cooperativo a través de la información gráfica y escrita proporcionada por distintos medios de comunicación.

La muestra está compuesta por 25 alumnos del Máster Oficial en Dirección de Empresas (MBA) de la Facultad de Economía de la UV. El perfil de nuestro alumnado es un tanto heterogéneo pero mayormente constituido por titulados en distintas ingenierías y en consecuencia, desconocen el campo de la Economía Financiera.

El recurso (medio de comunicación) seleccionado para llevar a cabo la innovación es el periódico "El Economista" porque, bajo nuestro punto de vista, en muchas de sus noticias utiliza elementos gráficos que son bastante pedagógicos, además de insertar algunos ejemplos. Esta característica nos parece muy interesante desde el punto de vista académico.

En la Tabla 1 detallamos las etapas en que se disgrega el diseño y desarrollo de esta actividad, cuyos contenidos tratan aspectos relacionados con la política de endeudamiento:

\section{Tabla 1. Etapas de la actividad}

I. Sellección de una Noticia Relacionada con Política de Endeuidamiento

La noticia que seleccionamos inicialmente trata sobre la política de endeudamiento que está acometiendo la cotizada Abengoa. Publicamos en "Aula Virtual" la primera noticia (véase Anexo I). Automáticamente después se informa de ella al alumnado y se les aconseja que lean con detenimiento en su casa el contenido de la noticia para que hagan todas aquellas anotaciones que consideren importantes a la hora de aclarar sus dudas.

Previo a la publicación de esta noticia no se les ha explicado a los alumnos ningún contenido al respecto de que efectos pueda tener la política de endeudamiento que una empresa pueda acometer, ni tampoco el significado de la jerga financiera utilizada. Tampoco se ha proporcionado material a nivel instrumental que explique estos contenidos.

II. Resolución de Dudas IniCiales Y "Clase Magistral"

En esta fase los estudiantes se organizan por grupos y debaten sobre sus respectivas lecturas a colación de la noticia anterior. Después, plantean al profesor todos sus comentarios y dudas poco relevantes sin entrar en detalles. Finalmente, el profesor explica aquellos contenidos fundamentales, como son:

- La emisión de obligaciones como fórmula de financiación a largo plazo para las firmas cotizadas.

- Elementos característicos de la emisión de deuda, etc.

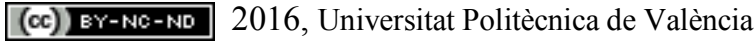

Congreso In-Red (2016) 
- Consecuencias en el valor cotizado por la recompra de bonos para ser amortizados.

- Efectos de la emisión de obligaciones convertibles.

- Efectos de los cambios en la política de endeudamiento actual.

Esta etapa se caracteriza por invertir el procedimiento docente tradicional. Por regla general el profesor transmite los conocimientos teóricos (clase magistral) y posteriormente, desarrolla ejercicios y ejemplos prácticos para ilustrar la teoría. Aquí estamos haciendo todo lo contrario: el alumno lee la noticia sin tener conocimientos previos y con posterioridad el profesor explica el significado de los elementos teóricos clave.

III. ANálisis de OTras Notictas Rblacionadas

Aquí el cometido del profesor es proporcionar en la plataforma virtual más noticias relacionadas con la misma temática publicadas por la misma entidad u otras. Cada una de esas noticias (véase Anexo II) implica la resolución de ejercicios prácticos cortitos y contestar a preguntas relativamente abiertas.

Este planteamiento, no tiene como finalidad hacer cálculos sino que sean capaces de interpretar adecuadamente y tomar decisiones en base a la información que se va proporcionando, así como fomentar el debate y la crítica constructiva.

IV. Análisis y Evaluación de las Competendias Profesionales

A través de la escala de Likert se evalúa en qué medida se han alcanzado las competencias profesionales con la experiencia descrita. El profesor elabora una plantilla donde aparecen escritas las competencias citadas en el apartado 3. La valoración la realizarán los alumnos a uno de sus compañeros dentro del mismo grupo, siendo el evaluado desconocedor del compañero que lo evalúa.

El profesor a su vez, también la cumplimenta la plantilla en base a la observación directa y a los resultados de los cuestionarios realizados en clase. La valoración final está compuesta por la media aritmética simple de ambas valoraciones (alumnos y profesor).

Para acabar, se comparan los resultados obtenidos en la prueba de evaluación final con los de los varios cursos inmediatamente anteriores.

\section{Resultados}

Procedemos a analizar los resultados de evaluación de los ítems correspondientes a las competencias profesionales proporcionadas en el apartado 3. De este análisis podemos concluir que:

(i) En términos generales la experiencia ha sido muy productiva a la par que satisfactoria tanto por parte del alumnado como del profesorado. 
(ii) La "Habilidad para analizar y buscar información proveniente de fuentes diversas (CG6)" y la "Capacidad para trabajar en equipo (CG12)", se enmarcan como las competencia profesionales con la valoración más elevada. Prácticamente toda la puntuación se encuentra concentrada en los valores más grandes (4 y 5 puntos).

(iii) En cambio, "Ser capaz de elaborar e interpretar la información contable, tanto la financiera, destinada a los usuarios externos, como la interna, destinada al control de gestión y la toma de decisiones (CE2)"; "Ser capaz de resolver problemas de valoración financiera, tanto en decisiones de financiación, como de inversión empresarial, en el entorno nacional e internacional (CE4)"; y finalmente, "Conocer los diferentes instrumentos de financiación y ser capaz de determinar la política de endeudamiento de la empresa (CE56)"; son las competencias profesionales, que aun obteniendo una valoración aceptable (entre 2 y 5 puntos), todavía deben mejorar.

(iv) La comparativa realizada entre los resultados respecto a los varios cursos académicos precedentes, queremos destacar que para las cuestiones referentes a políticas de endeudamiento, han sido muy parecidas en las tres convocatorias y por tanto, comparables. En este caso el procedimiento comparativo se realiza exclusivamente con los resultados referentes a los conocimientos adquiridos, no al nivel de adquisición de competencias profesionales. Éstos nos revelan que las calificaciones tras aplicar esta metodología han alcanzado cotas más altas.

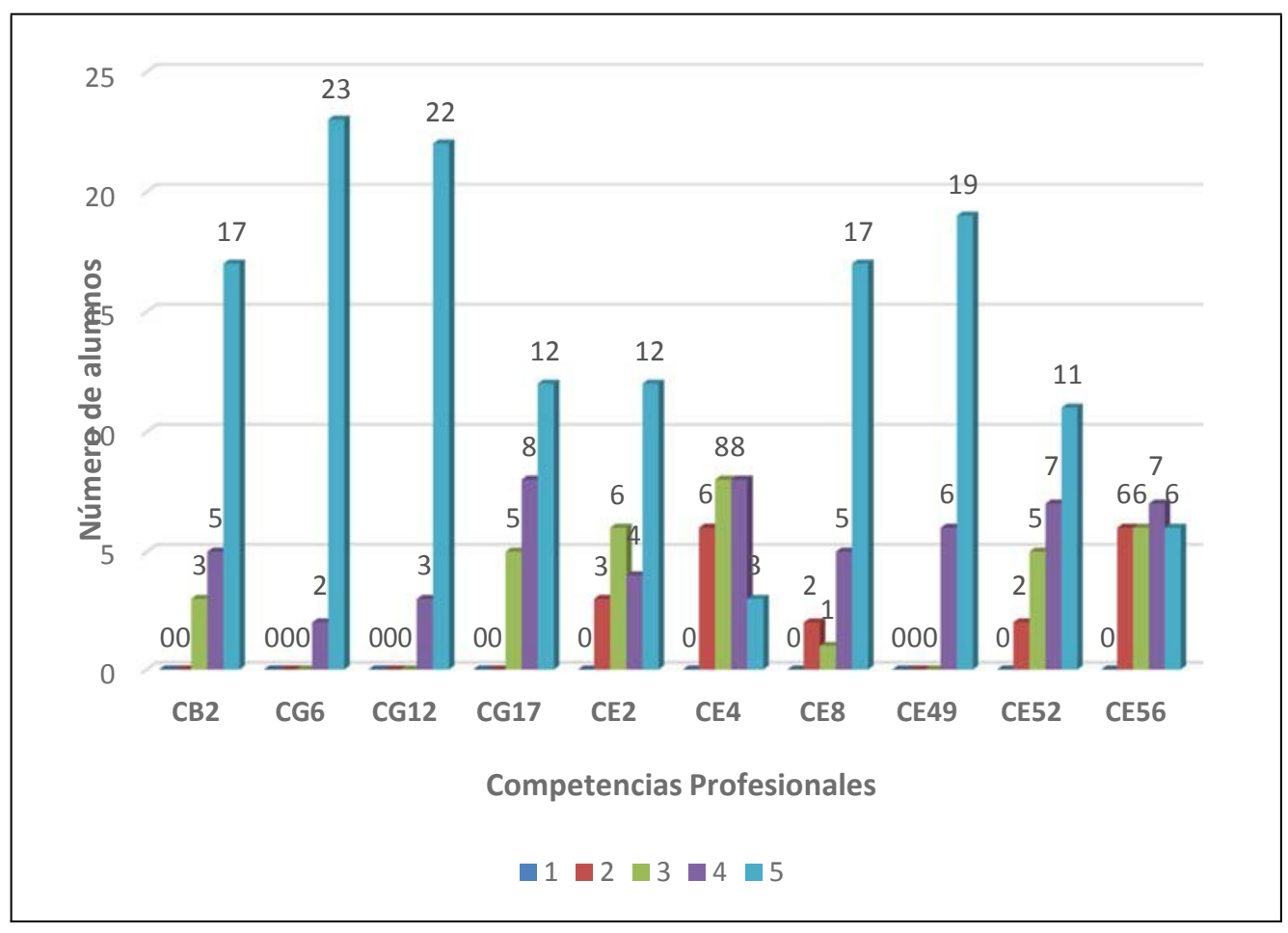

Gráfico 1. Resultados de evaluación de competencias profesionales 
En términos cuantitativos, la media de las calificaciones del grupo asciende en un 12,85\% mayor respecto al curso anterior y de un 11,34\% mayor respecto a dos cursos anteriores.

Basándonos en la experiencia desarrollada, en los comentarios vertidos en el aula y en las preguntas formuladas al respecto por el profesorado, apreciamos que nuestros alumnos han hecho una valoración muy positiva de los recursos inherentes en la prensa gráfica, lo cual deja de manifiesto los aspectos beneficiosos derivados de la experiencia desarrollada en el aula. Estas opiniones se suman a la valoración positiva por parte del docente a la hora de evaluar la participación de sus estudiantes y el desarrollo de las competencias profesionales.

\section{Conclusiones}

El objetivo fundamental de la experiencia que presentamos en este trabajo es formar en competencias profesionales a los alumnos que cursan el Máster de Dirección de Empresas (MBA) de la Facultad de Economía (UV). Para ello hemos implementado una metodología de Trabajo Cooperativo como vehículo para transmitir los conocimientos de forma activa, participativa, crítica y constructiva. A nuestro juicio, estamos convencidos que el método bien implementado podría ser una buena opción para valorar en qué medida los alumnos están desarrollando dichas competencias que el currículo de su titulación exige, mediante el estudio y análisis de la información contenida en la prensa gráfica de carácter financiero.

El diagnóstico de la innovación a través de la evaluación de la experiencia así como del rendimiento académico señala como muy idóneo el método implementado, así como los medios de comunicación de prensa gráfica. Efectivamente, los resultados de evaluación de las competencias profesionales nos permiten aseverar que, generalmente, en gran parte de los ítems la valoración se encuentra en un intervalo muy próximo a la máxima calificación, y en consecuencia la asimilación de las competencias profesionales muestra niveles más que aceptables. Estos avances además se materializan en los resultados académicos ya que, la calificación media del curso presente respecto a los dos cursos precedentes, nos revelan una mejora del $12,85 \%$ y $11,34 \%$, respectivamente.

\section{Referencias}

ARIZA, A. (2000). Las Nuevas Tecnologías de la Información y la Comunicación y una Propuesta para el Trabajo Colaborativo. V Congreso Iberoamericano de Informática Educativa. http://www.edudistan.com/Adolfo\%20Ariza.htm [Consulta: 5 de octubre de 2015]

ARNOLDI, J. (2007). "Universities and the public recognition of expertise" en Minerva, vol. 45, issue 1, p. 49-61.

BUIL, I., HERNÁNDEZ, B. y SESÉ, F.J. (2013). "El papel de la prensa en la mejora del proceso de aprendizaje. Una aplicación en el EEES" en Revista de Docencia Universitaria. $R E D U$, vol. 3, p. 445-459.

CABERO, J. y GUERRA, S. (2011). "La alfabetización y formación en medios de comunicación en la formación inicial del profesorado (media literacy in the initial teacher education)" en Educación XX1, vol. 14, issue 1, p. 89-115.

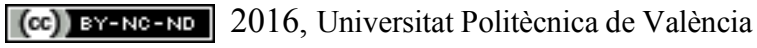


DE ARIAS, M.E. (2005). "Sobre el para qué de la comunicación social: aproximación a la teoría de la mediación” en Anuario Ininco, vol 7, issue 2, p. 375-395.

GUTIÉRREZ, J.J., RODRÍGUEZ, M., BELTRÁN, J.F. y CONRADI, M. (2013) Zoowiki: un Proyecto de colaboración Interdepartamental. I jornada de Innovación Educativa. Facultad de Ciencias de la Educación. Universidad de Sevilla. https://fcce.us.es/sites/default/files/docencia/MESA6pdf/5.pdf [Consulta: 18 de septiembre de 2015]

HEIJKE, H., MENG, C. y RIS, C. (2003). "Fitting to the Job: The Role of Generic and Vocational Competencies in Adjustment and Performance" en Labour Economics, vol. 10, p. 215-229.

LAYER, G. (2004). Widening Participation and Employability. York: Learning and Teaching Support Network.

MCCLUNE, B. y JARMAN, R. (2010). "Critical reading of science-based news reports: Establishing a knowledge skills and attitudes framework" en International Journal of Sciencie Education, vol. 32, issue 6, p. 727-752.

MUÑOZ, F., ARVAYO, K., VILlEGAS, C., GONZÁlEZ, F. y SOSA, O. (2014). "El método colaborativo como una alternativa en el trabajo experimental de Química Orgánica" en Educación Química, vol. 25, issue 4, p. 464-469.

RICOY, M.C. (2006). "La prensa como recurso en el curriculum de adultos" en Comunicar, vol. 14, issue 26, p. 199-206.

SÁEZ, J. y RUÍZ, J.M. (2012). "Metodología didáctica y tecnología educativa en el desarrollo de las competencias cognitivas: aplicación en contextos universitarios" en Revista de Currículum y Formación del Profesorado, vol. 16, issue 3, p. 373-391.

SEMEIJN, J.H., VELDEN, R.K.., HEIJKE, J.A.., VLEUTEN, C.P. y BOSHUIZEN, H.C. (2005). Competence Indicators in Academic Education and Early Labour Market Success of Graduates in Health Sciences. Maastricht: Research Centre for Education and the Labour Market. 
Los medios de comunicación como recurso para formar en competencias en el área de la Economía Financiera

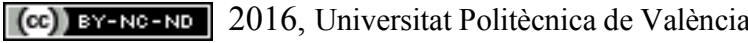

Congreso IN-RED (2016) 
Anexo I. Noticia de política de endeudamiento de Abengoa

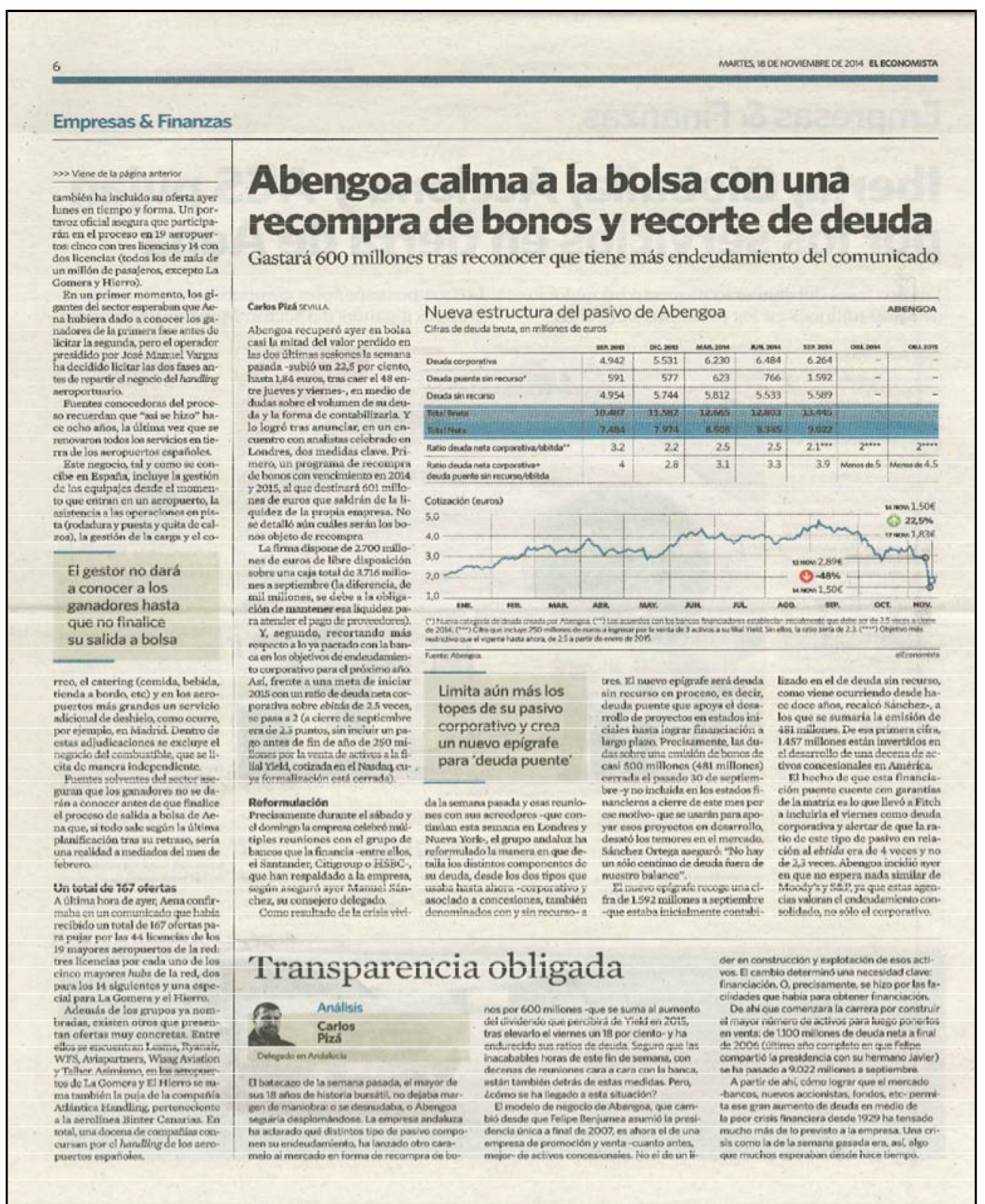

Fuente: www.eleconomista.es 
Anexo II. Noticia de política de endeudamiento de Acciona

$$
18
$$

\section{Bolsa \& Inversión}

\section{Acciona emite convertibles para bajar su coste de financiación del $5,6 \%$ al $3 \%$}

Captará 325 millones con deuda canjeable por En el peor de los casos el grupo debería repartir acciones que la compañía 'valora' a 63.02 euros acciones por valor de un $9 \%$ del capital actual

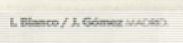

Ei nafio llega curgido de entrenos

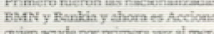

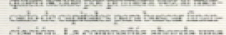

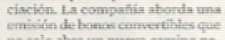

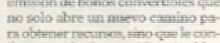

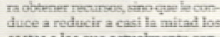

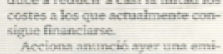

Acelons ant

que captarí al memos 325 mithere

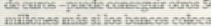

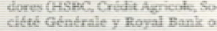

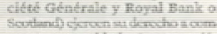

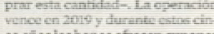

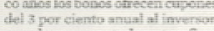

Papderos semetrinchente a fint:

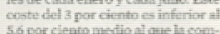

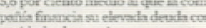

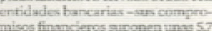

Eses al bereficio bruts

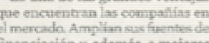

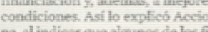

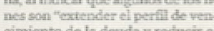

coste medio de financiectitn, a:

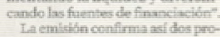

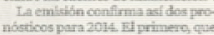

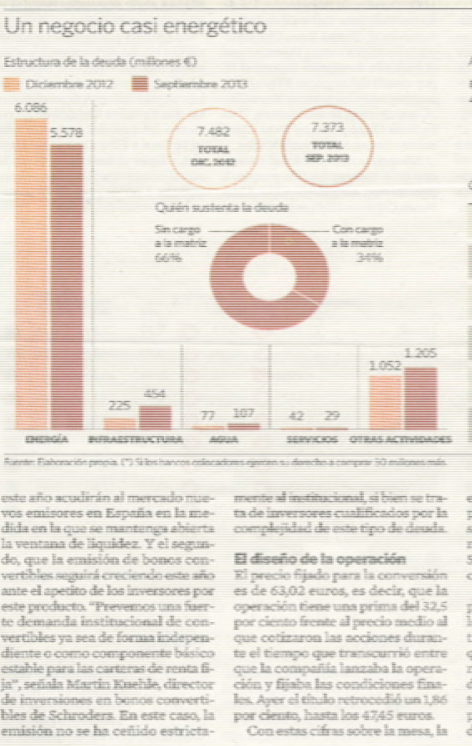

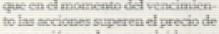

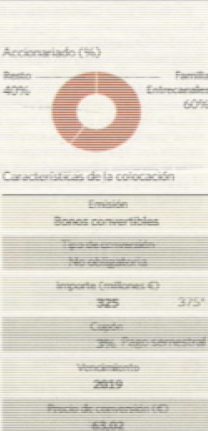

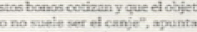

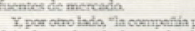

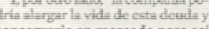

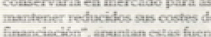

Es, con lo eque de ninguna manes?

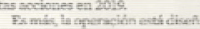

da de forma que, sunque en ef

Cocerse una ampliackn nis sicos

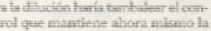

La familla

Entrecanales no

perderia el control

de la companía

enningun

familis Entreamales, cuya partic.

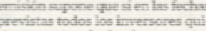

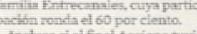

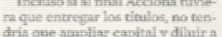

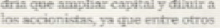

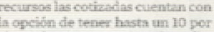

Fis lo que recurrib, per ejem.

ine hetreste linite de matocart

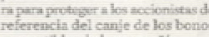

Fuente:www.eleconomista.e 